\title{
Cannabis Use among People with Mental Illness: Clinical and Socio-Demographic Characteristics
}

\author{
Sumia A. Al Azizi' ${ }^{1}$ Abdelaziz A. Omer², Amir A. Mufadde ${ }^{3 *}$ \\ ${ }^{1}$ Sudan Medical Specialization Board, Khartoum, Sudan \\ ${ }^{2}$ Department of Psychiatry, University of Khartoum, Sudan \\ ${ }^{3}$ Department of Psychiatry, Mediclinic Al Jawhara Hospital, Al Ain, United Arab Emirates \\ Email: *khalifaamir@yahoo.co.uk
}

How to cite this paper: Al Azizi, S.A., Omer, A.A. and Mufaddel, A.A. (2018) Cannabis Use among People with Mental Illness: Clinical and Socio-Demographic Characteristics. Open Journal of Psychiatry, 8, 244-252.

https://doi.org/10.4236/ojpsych.2018.83021

Received: April 4, 2018

Accepted: July 7, 2018

Published: July 10, 2018

Copyright $\odot 2018$ by authors and Scientific Research Publishing Inc. This work is licensed under the Creative Commons Attribution International License (CC BY 4.0).

http://creativecommons.org/licenses/by/4.0/

\begin{abstract}
Background: Cannabis can be associated with short-term and long-term adverse effects such as psychotic states, risk of dependence, provoking relapse and aggravating existing psychotic symptoms. Aim: The current study is a descriptive cross-sectional hospital based study that aims to identify the prevalence and correlates of cannabis use among patients with mental illness. Methods: The study was conducted in two outpatient psychiatry clinics in Khartoum (Sudan). The sample size was 348 consecutive patients over 2 months' study period. Clinical and sociodemographic characteristics were documented. Descriptive statistics was used to describe the data variables, and tabulated tables were used to calculate chi-square test for categorical data and the significance was set at $p<0.05$. Results: A total of 96 participants (27.6\%) had history of cannabis abuse; all of them were male patients. The majority (51\%) started to use cannabis between 10 - 19 years of age, and most of them (86.6\%) had a duration of less than five years of using cannabis. Psychiatric symptoms occurred after starting cannabis use in $17.7 \%$ of cases. We found a statistically significant difference related to age when comparing the age of cannabis users and non-users $(p=0.0239)$. The majority of patients with comorbid mental illness and cannabis abuse (89.5\%) were below the age of 40 years. There was also statistically significant association between educational level achieved and history of using cannabis $(p=0.00001)$. Those with history of cannabis are more likely to be manual laborers (46.9\%), and those with no history of cannabis were mostly unemployed (44\%), indicating significant influence of employment history $(\mathrm{p}=0.0064)$. The relation between using cannabis and the clinical psychiatric diagnosis was not statically significant $(\mathrm{p}=0.125)$. Conclusion: Cannabis use is highly prevalent among people with mental illness. It is related to age, educational level and type of work.
\end{abstract}




\section{Keywords}

Cannabis, Sociodemographic, Psychiatric Disorders, Schizophrenia, Mood Disorders

\section{Introduction}

Cannabis is the most commonly consumed illegal drug and self-reported consumption has continued to grow through the 1990s. There is controversy around the reported rise in its use but also little clarity about what has driven this rise. The health effects of cannabis range from discussion on the long-term health effects of cannabis to the debate on the potential therapeutic benefits of cannabinoids in some medical conditions [1].

Known and valued for centuries as a euphoriant, cannabis (bango, marijuana, charas, hashish) was extensively used in the nineteenth century as an analgesic, anticonvulsant and hypnotic. Recent studies indicate that its derivatives may be useful in the treatment of glaucoma and nausea produced by cancer chemotherapy [2] [3]. On the other hand, a considerable proportion of cannabis users report adverse effects related to cannabis use, such as psychotic states and risk of dependence. People with major psychiatric disorders including schizophrenia are more vulnerable to developing adverse effects as cannabis can provoke relapse and aggravates existing psychotic symptoms [4].

Studies on patients who discontinued cannabis have shown significant improvement in both physical and psychiatric clinical features. For example, a Dutch cohort study has shown that patients who discontinued their cannabis use after the first baseline assessment had a greater increase in their body mass index, waist circumference, and diastolic blood pressure and triglyceride concentrations than other patients. The severity of their psychotic symptoms had decreased more compared to those who continued cannabis use [5]. The limited data at present suggests that heavy cannabis use is more evident among indigenous populations and that up to one in two cannabis users in remote communities experience adverse effects related to mental health [6].

\section{Aim}

The current study is a descriptive cross-sectional hospital based study that aims to identify the magnitude and nature of cannabis dependence among patients with mental illness and their motivation and readiness to quit in two outpatient psychiatry clinics in Khartoum state-Sudan. Specific aims are to estimate prevalence of cannabis use among patients with psychiatric illness and to identify possible associated factors.

\section{Methods}

The study was conducted in two psychiatric hospitals in Khartoum including 
Taha Baasher Hospital and El-tigani El-Mahi Hospital. The study was conducted in outpatient settings, no inpatient included.

Taha Bassher Hospital was the first established psychiatric clinic in Sudan that has been providing its outpatient psychiatric services since 1962 and then changed to be a hospital. The hospital is located in Khartoum North area of Khartoum state in Sudan. The hospital serves more than 1500 patients monthly in different departments (Outpatient, in patient and emergency unit). The total hospital capacity is 61 with bed occupancy estimates at 94.7\%. El-Tigani El-Mahi Hospital took the name of the first African Psychiatrist [7], and its full range of inpatient services started in 1971 as a national psychiatric hospital. It is located in Omdurman area of Khartoum state. The hospital serves more than 3000 patients monthly in different departments (Out-patient, inpatient and emergency unit). The total hospital capacity is 120 beds with bed occupancy of $97.2 \%$.

The study sample include all the patients who were attending the outpatient clinic of Taha Bassher Hospital (on Sundays and Tuesdays) and El-Tigani El-Mahi Hospital on (Mondays and Wednesdays) during the 2 months' study period. The study excluded patients with mental retardation, patients with severe cognitive dysfunction and patients who are less than 18 years. The sample size was 348 consecutive patients. Descriptive statistics was used to describe the data variables, and tabulated tables were used to calculate chi-square test for categorical data and the significance was set at $p<0.05$. The study was approved by Sudan Medical Specialization Board (SMSB) and the related hospitals.

\section{Results}

The study included a total of 348 participants (263 males and 85 females) who had history of mental illness. A total of 96 participants (27.6\%) had history of cannabis abuse, all of them were male patients.

Table 1 summarizes the clinical and socio-demographic characteristics of patients with mental illness who had history of cannabis abuse. The majority (51\%) of users started to use cannabis between 10 - 19 years of age. Only 3.1\% reported that they started using cannabis after the age of 30 years. Most of the cannabis users $(86.6 \%)$ had a duration of less than five years of using cannabis. The development of psychiatric symptoms occurred after starting the use of cannabis in $17.7 \%$ of cases. The consumption is of cannabis tends to be increased by users as reported by $35.4 \%$ of participants, and it has effect on daily activities that is reported by $57.3 \%$ of them. The majority $(84.4 \%)$ reported their desire to stop using cannabis, and more than $90 \%$ had at least one previous trial to quit with $41.7 \%$ reporting previous history of withdrawal symptoms. Factors that limit the participants' efforts to quit were boredom (38.5\%), stress and anxiety (33.3\%), habituation (18.8\%) and other psychological symptoms (9.4\%) (Table 1).

We compared people with mental illness who used cannabis and those who do not based on age, psychiatric diagnosis, occupational status, marital history, and 
educational level. We found a significantly statistical difference related to age of participants when comparing the age of cannabis users and non-users $(p=$ 0.0239). The majority of mentally ill patients with history of cannabis abuse (89.5\%) were below the age of 40 years of whom there were $45.8 \%$ below the age of 30 years (Table 2).

Figure 1 shows the association between cannabis use and the type of psychiatric diagnosis compared with those who have no history of cannabis. The rate of schizophrenia was the most common psychiatric diagnosis in both groups and it was particularly higher in psychiatric patients with history of cannabis (43.7\%) than those with no history of using cannabis (38.5\%). However, the relation between using cannabis and the clinical psychiatric diagnosis was not statically significant $(p=0.125)$.

Figure 2 shows statistically significant association between employment status when comparing people with mental illness who have history of cannabis abuse and those who haven't $(\mathrm{p}=0.0064)$. Those with history of cannabis are more likely to be manual laborers (46.9\%), and those with no history of cannabis were mostly unemployed (44\%).

There was statistically significant association between educational level achieved and history of cannabis abuse in people with mental illness ( $\mathrm{p}=$ $0.00001)$, Table 3. Most of the cannabis users had level of education that is below the secondary school level (65.6\%), those who had secondary school level were $24 \%$ and only $10.4 \%$ were university graduate. Those who have no history of cannabis, had better educational achievement with $18.3 \%$ who were university graduates and $25.8 \%$ who had secondary school education.

The vast majority of both cannabis users (67.7\%) and non-users (55.6\%) were single with no statistically significant difference between the two groups despite the increased number of single individuals who are using cannabis $(p=0.0559)$. Number of married individuals is slightly higher in those who are not using cannabis (Table 4).

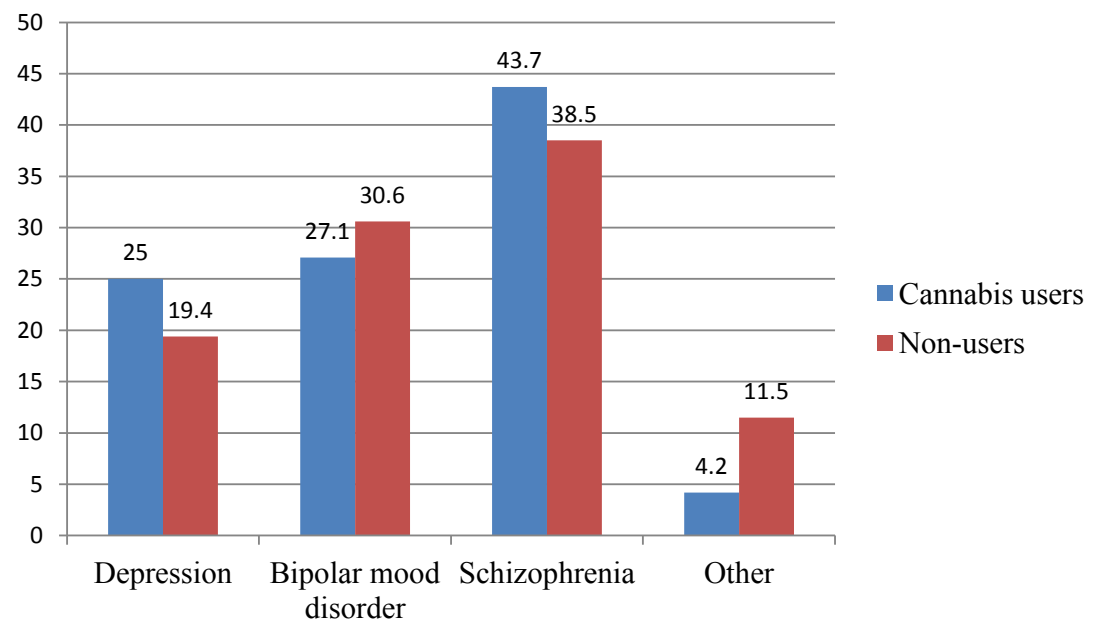

Figure 1. Cannabis users versus non-users: Distribution according to psychiatric diagnosis. Chi-square $=5.7381 . \mathrm{P}=0.1250$. The result is not significant at $\mathrm{p}<0.05$. 


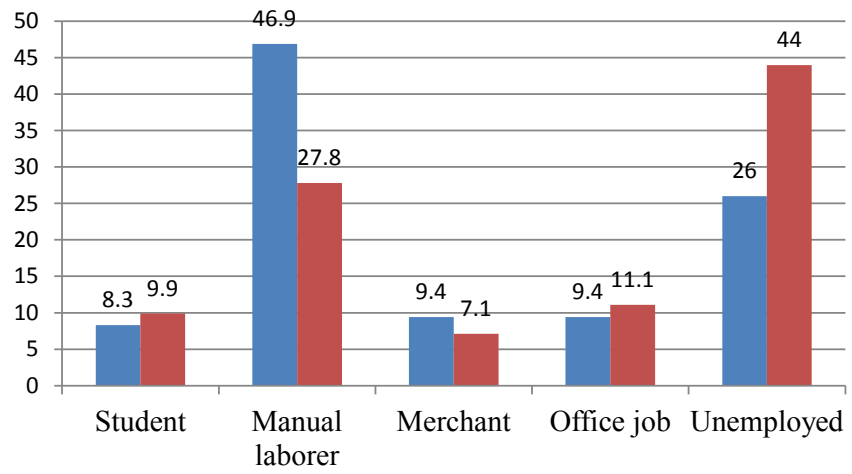

- Cannabis users

- Non-users

Figure 2. Cannabis users versus non-users: Distribution according to employment status. The chi-square $=14.2675, \mathrm{p}=0.0064$. The result is significant at $\mathrm{p}<0.05$.

Table 1. Clinical and socio-demographic characteristics.

\begin{tabular}{|c|c|c|}
\hline & Frequency & $\%$ \\
\hline \multicolumn{3}{|l|}{ Age at starting cannabis use: } \\
\hline $10-19$ years & 49 & 51 \\
\hline $20-29$ years & 44 & 45.8 \\
\hline 30 years or more & 03 & 03.1 \\
\hline \multicolumn{3}{|l|}{ Duration of using cannabis } \\
\hline Less than 5 years & 86 & $89.6 \%$ \\
\hline $5-10$ years & 07 & 7.3 \\
\hline More than 10 years & 03 & 3.1 \\
\hline \multicolumn{3}{|c|}{ Cannabis use preceding psychiatric symptoms: } \\
\hline Yes & 17 & 17.7 \\
\hline No & 79 & 82.3 \\
\hline \multicolumn{3}{|l|}{ Increasing amount of smoked cannabis } \\
\hline Yes & 34 & 35.4 \\
\hline No & 62 & 64.6 \\
\hline \multicolumn{3}{|l|}{ Effect of cannabis on daily activities } \\
\hline Yes & 55 & 57.3 \\
\hline No & 41 & 42.7 \\
\hline \multicolumn{3}{|l|}{ Desire to continue using cannabis } \\
\hline Yes & 15 & 15.6 \\
\hline No & 81 & 84.4 \\
\hline \multicolumn{3}{|l|}{ History of withdrawal symptoms } \\
\hline Yes & 40 & 41.7 \\
\hline No & 56 & 58.3 \\
\hline \multicolumn{3}{|l|}{ Trials to quit: } \\
\hline Never & 7 & 7.3 \\
\hline Once & 55 & 57.3 \\
\hline $2-3$ trials & 29 & 30.2 \\
\hline More than 3 trials & 5 & 5.2 \\
\hline \multicolumn{3}{|l|}{ Barriers to quit } \\
\hline Boredom & 37 & 38.5 \\
\hline Stress and anxiety & 32 & 33.3 \\
\hline Habituation & 18 & 18.8 \\
\hline Other psychological symptoms & 9 & 9.4 \\
\hline
\end{tabular}


Table 2. Cannabis users versus non-users: distribution according to age.

\begin{tabular}{ccc}
\hline Age group & Cannabis users: $\mathrm{n}(\%)$ & Non-users: $\mathrm{n}(\%)$ \\
\hline $19-29$ years & $44(45.8 \%)$ & $108(42.9 \%)$ \\
$30-39$ years & $41(42.7 \%)$ & $83(32.9 \%)$ \\
40 years and above & $11(11.5 \%)$ & $61(24.2 \%)$ \\
Total & $96(100 \%)$ & $252(100 \%)$ \\
\hline
\end{tabular}

The chi-square $=7.4643 . \mathrm{p}=0.0239$. The result is significant at $\mathrm{p}<0.05$.

Table 3. Cannabis users versus non-users: distribution according to educational level.

\begin{tabular}{ccc}
\hline & Cannabis users: $\mathrm{n}(\%)$ & Non-users: $\mathrm{n}(\%)$ \\
\hline Illiterate & $4(4.2 \%)$ & $57(22.6 \%)$ \\
Informal education & $20(20.8 \%)$ & $23(9.1 \%)$ \\
Basic level & $39(40.6 \%)$ & $61(24.2 \%)$ \\
Secondary school level & $23(24 \%)$ & $65(25.8 \%)$ \\
University education & $10(10.4 \%)$ & $46(18.3 \%)$ \\
Column Totals & $96(100 \%)$ & $252(100 \%)$ \\
\hline
\end{tabular}

The chi-square $=30.4809 . \mathrm{P}<0.00001$. The result is significant at $\mathrm{p}<0.05$.

Table 4. Cannabis users versus non-users: distribution according to marital status.

\begin{tabular}{ccc}
\hline & Cannabis users: $\mathrm{n}(\%)$ & Non-users: $\mathrm{n}(\%)$ \\
Single & $65(67.7)$ & $140(55.6)$ \\
Married & $27(28.1)$ & $85(33.7)$ \\
Separated/widowed & $04(4.2)$ & $27(10.7)$ \\
Column Totals & $96(100)$ & $252(100)$ \\
\hline
\end{tabular}

The chi-square $=5.7671$. The $\mathrm{p}$-value $=0.0559$. The result is not significant at $\mathrm{p}<0.05$.

\section{Discussion}

Cannabis is one of the most common substances of abuse in Sudan. A recent epidemiological study in the country has shown a higher life time prevalence of cannabis use than alcohol use among university students. The latter study estimated the current prevalence of various substances used by university students including tobacco (13.7\%), cannabis (4.9\%), alcohol (2.7\%), amphetamines (2.4\%), tranquilizers $(3.2 \%)$, inhalants (1\%), opiates $(1.2 \%)$, cocaine $(0.7 \%)$, and heroin use $(0.5 \%)$ [8].

The present study revealed that the prevalence of cannabis use among mentally ill patients is $27.6 \%$ which is a considerable percentage indicating the need of more surveys and research from campaign against drug use. Study which assessed cannabis use among psychiatry outpatients with depression (at baseline and at 6 months of treatment), has shown that $40.7 \%$ of patients with depression used cannabis when assessed at baseline which then decreased significantly over 6 months of treatment. The study also revealed that cannabis has significantly 
worsened the symptoms of depression and led to poorer mental and physical functioning [9].

One of the gaps in cannabis research that were identified in the World Health Organization (WHO) report on the health effects of cannabis was the lack of epidemiological data on patterns and consequences of cannabis use in developing countries [10]. The variable prevalence rates of using cannabis among psychiatric and normal population could be attributed to multiple factors, most importantly, research sample characteristics, and methodological differences in different studies, genetic vulnerability, personality dynamics and socio-cultural affiliations.

The study disclosed that all cannabis using were males (100\%), while females with mental illness reported no use cannabis. But this not a revelation if we knew that cannabis using for females has some cultural restriction in this country. It has been reported that with increasing age, using frequently tends to be less, and this was also observed in this study (i.e., the majority of those who are using cannabis were between 19 - 29 years of age). It is, therefore, logical for campaigns against drug use to target young adult psychiatric patients who are the victims of multiple complex problems. Most of the cannabis users started using the substance during adolescence and at age group that is vulnerable to developing mental illness. This can be explained by so many factors such as curiosity, impulsive behavior and vulnerability which lead this age group to explore new experiences. Emergence of psychiatric symptoms might be induced by cannabis or can be also explain the maintenance of the behavior of using cannabis in order to overcome the anxiety or stress in some of them as reported by over $30 \%$ of participants.

Regarding the socio-demographic factors, the study disclosed that using inversely related to educational level, age and type of employment. No statistically significant relation between cannabis use and the marital status despite the finding that over sixty percent of cannabis users were single. The latter findings, are consistent with other studies in most of the investigated variables. For example, an Australian study that was conducted among mentally ill patients has found association between cannabis dependence and the following factors: being male, younger age (18 - 24), and being unemployed, but no relationship was found between level of education and cannabis dependence [11]. This slight variation can be explained by cultural background and economical differences across various societies.

We found diagnosis of schizophrenia is the most common among cannabis users, followed by bipolar mood disorder and depression. The type of mental illness per say is not a significant factor related to cannabis dependence. There is consistent evidence from cross-sectional research that cannabis and psychotic disorders, particularly schizophrenia, occur together more often than would be expected by chance and those in treatment for psychosis have high rates of cannabis use disorders [12] [13] [14]. 
Consistent with other studies, we found $17 \%$ of patients reporting emergence of psychiatric symptoms following cannabis use. Other studies revealed high risk of developing psychosis induced by cannabis and supporting a causal link between cannabis and psychotic symptoms [15].

Although there was complexity involved in measuring motivation, the survey questions in our study found the majority of participants cited psychological symptoms and other psychosocial stressors as barriers to quit. Addressing social economic, and employment factors and the related stress issues as well as providing sufficient support can be effective majors towards prevention strategies. Enhancing motivation among those who have readiness to quit and promoting factors that improve motivation are also important the majority of participants had previous trials to stop taking cannabis. Previous studies disclosed that cannabis users particularly adolescents shown low motivation for change though experiencing more problems associated with its use emerges as a determinant of increased motivation [16]. Previous research on other types of dependence in the same study area (Sudan), has also shown an increased desire to quit, and multiple but failed trials of abstinence in patients with alcohol dependence. Participants from the latter study had longer duration of using alcohol than that reported by patients using cannabis in the current study. However, they s were also likely to be male patients of less than 35 years (54\%), and single (54\%) [17]. Therefore, efforts in reducing drug related problems (cannabis and alcohol) in the study area should be focused on individuals who have these particular characteristics. Raising awareness, social problems solving and specialized centers can be part of the preventive measures.

\section{Conclusion}

Cannabis use is highly prevalent among people with mental illness. It is related to age, educational level and type of work. No significant relation between the psychiatric diagnosis and cannabis use. Integrated, combined, and evidence-based psychosocial and pharmacological interventions are required within mental health and addiction treatment settings to improve quit success.

\section{Conflict of Interest}

The authors declare no conflict of interest.

\section{References}

[1] Farrell, M. and Ritson, B. (2001) Cannabis and Health. British Journal of Psychiatry, 178, 98. https://doi.org/10.1192/bjp.178.2.98

[2] Bostwick, J.M. (2012) Blurred Boundaries: The Therapeutics and Politics of Medical Marijuana. Mayo Clinic Proceedings, 87, 172-186.

https://doi.org/10.1016/j.mayocp.2011.10.003

[3] Chaudhury, H.R. and Brig, S. (2005) Cannabis Use in Psychiatric Patients. MJAFI, 61, 117-120.

[4] Johns, A. (2001) Psychiatric Effects of Cannabis. British Journal of Psychiatry, 178, 
116-122. https://doi.org/10.1192/bjp.178.2.116

[5] Bruins, J., Pijnenborg, M.G., Bartels-Velthuis, A.A., et al. (2016) Cannabis Use in people with Severe Mental Illness: The Association with Physical and Mental Health-A Cohort Study. A Pharmacotherapy Monitoring and Outcome Survey Study. Journal of Psychopharmacology, 30, 354-362. https://doi.org/10.1177/0269881116631652

[6] Arsenault, L., Cannon, M., Poulton, R., et al. (2002) Cannabis Use in Adolescence and Risk for Adult Psychosis: Longitudinal Prospective Study. British Medical Journal, 325, 1212-1213. https://doi.org/10.1136/bmj.325.7374.1212

[7] Omer, A.A. and Mufaddel, A.A. (2018) Attitudes of Patients with Psychiatric Illness toward Traditional Healing. International Journal of Social Psychiatry, 64, 107-111. https://doi.org/10.1177/0020764017748987

[8] Osman, T., Victor, C., Abdulmoneim, A., et al. (2016) Epidemiology of Substance Use among University Students in Sudan. Journal of Addiction, 2016, 2476164. https://doi.org/10.1155/2016/2476164

[9] Bahorik, A.L., Leibowitz, A., Sterling, S.A., et al. (2017) Patterns of Marijuana Use among Psychiatry Patients with Depression and Its Impact on Recovery. Journal of Affective Disorders, 213, 168-171. https://doi.org/10.1016/j.jad.2017.02.016

[10] WHO Expert Working Group on Health Effects of Cannabis Use (1997) Cannabis: A Health Perspective and Research Agenda. WHO, Geneva.

[11] Swift, W., Hall, W. and Teesson, M. (2001) Cannabis Use and Dependence among Australian Adults: Results from the National Survey of Mental Health and Wellbeing. Addiction, 96, 737-748. https://doi.org/10.1046/j.1360-0443.2001.9657379.x

[12] Agosti, V. and Nunes, F.L. (2002) Rates of Psychiatric Comorbidity among U.S. Residents with Lifetime Cannabis Dependence. American Journal of Drug and Alcohol Abuse, 28, 643. https://doi.org/10.1081/ADA-120015873

[13] Grech, A., Van Os, J., Jones, P.B., et al. (2005) Cannabis Use and Outcome of Recent Onset Psychosis. European Psychiatry, 20, 349-53. https://doi.org/10.1016/j.eurpsy.2004.09.013

[14] Thomas, H.A. (1996) Community Survey of Adverse Effects of Cannabis Use. Drug and Alcohol Dependence, 42, 201-207. https://doi.org/10.1016/S0376-8716(96)01277-X

[15] Arseneault, L., Cannon, M., Witton, J., et al. (2004) Causal Association between Cannabis and Psychosis: Examination of the Evidence. British Journal of Psychiatry, 184, 110-7. https://doi.org/10.1192/bjp.184.2.110

[16] Fernández-Artamendi, S., Fernández-Hermida, J.R., García-Fernández, G., et al. (2013) Motivation for Change and Barriers to Treatment among Young Cannabis Users. European Addiction Research, 19, 29-41. https://doi.org/10.1159/000339582

[17] Omer, A.A. and Mufaddel, A. (2016) Characteristics and Motivation to Treatment in Male Patients Admitted with Dependence on a Local Type of Alcohol in Sudan. Journal of International Research in Medical and Pharmaceutical Sciences, 7, 14-18. 